\title{
Lactancia Materna Exclusiva: Crecimiento del Lactante en un Grupo Seleccionado de Niños Chilenos
}

\author{
Dra. Gabriela Juez G., Dra, Soledad Díaz F., Dr. Octavio Peralta M., \\ Dr. Horacio B. Croxatto A. ${ }^{1}$ \\ Matr. María Eugenia Casado G., Matr. Ana María Salvattíerra M., \\ Matr. Iliana Durán G. ${ }^{2}$, A. Social: Miriarn Femánde ${ }^{3}$
}

Growth Pattem in Selected Chilean Breastfed Infants

\begin{abstract}
This report describes the growth pattern of healthy, low middle and low socioeconomic class Chilean infants during exclusive breastfeeding. 242 infants who were on exclusive breastfeeding at day 30 postpartum entered the study. Of these, $59 \%$ were fully nursing at 6 months and grew at a nommal rate without recciving etiher supplementary milk or non dairy food. Suppplementary feedings were administered in $27 \%$ of cases because of suspected primary inadequate mitk ouput and in $14 \%$ of cases for other reasons such as pregnancy, illness, matemal work of self prescription. Full nursing provided the highest rate of weight increase during the first 4 mon ths of life and a greater weiglt gain for boys than for girls up to the age of 6 months. Gastrointestinal pathology, malnourishment or hospitalization were rare events in this population. It is concluded that maternal milk alone, if produced in sufficient amounts, can maintain normal grow th up to the 6th month of life. The study supports the choice of exclusive breastfeeding on demand plus child growth monitoring up to six months over routine prescription of supplements at earlier times.
\end{abstract}

En los últimos 60 afios se ha observado un descenso progresivo en la práctica de lactancia natural en el mundo y en Chile ${ }^{1-2 \cdot 3 \cdot 4}$. Este fenómeno ha tenido múltiples consecuencias sobre la población. Para los países subdesarrollados, el abandono precoz de la lactancia materna ha significado efectos a corto plazo sobre la salud del nino menor, aumentando el riesgo de muerte, morbilidad, desnutrición y la frecuencia de hospitalizaciones ${ }^{1-2-4-5-6-7-8}$. Además es necesario considerar el elevado costo económico que representa la no producción masiva de leche humana y su reemplazo por leches artificiales de mayor costo de elaboración ${ }^{2 \cdot 4-8}$.

Estudios realizados en los paises desarroliados han sugerido tambjén efectos a largo plazo relacionados al abandono masivo de la lactancia, entre los que se han señalado la obesidad y las enfermedades coronarias en el hijo y el cáncer mamario en la mujer ${ }^{9}$.

Por estas razones, diversos investigadores $\mathrm{e}$ instituciones tanto chilenas como extranjeras se han preocupado de estudiar las causas del aban-

${ }^{1}$ Médicos Consultorio de Planificación Familiar, J.V. Lastarria 29, Dep. 11 San tiago.

2 Matronas Consultorio de Planificación Familiar, J.V. Lastarria 29, Dep. 11 Santiago.

3 Asístente social: Consultorio de Planificación Familiar. J.V. Lastarria 29, Dep. I I, Santiago. dono de la lactancia natural y de buscar medios de revertirlo. Se ha sostenido la hipótesis de que cabe una gran parte de responsabilidad al equipo de salud en la obtención de una lactancia exito$\mathrm{sa}^{2 \cdot 10-11-12-13 \cdot 14}$.

Por otra parte, se ha puesto en duda que la leche materna sea suficiente nutrición para el lactante mayor de 3 meses lo que ha llevado a recomendar la suplementación temprana ${ }^{15-16}$. Sin embargo, esta práctica conlleva el riesgo de la inadecuada preparación o calidad de los suplementos $y$ el consiguiente aumento en la tasa de enfermedades y desnutrición en aquellas regiones en que el costo de dicha alimentación afecta el presupuesto familiar ${ }^{2-4-17}$.

El objetivo de este estudio prospectivo fue observar el crecimiento del lactante sano, exclusivamente alimentado al pecho y bajo circunstancias controladas. En este estudio se prestó especial atención al manejo de la lactancia por el equipo de sálud y al control periódico del niño, para evitar cualquier riesgo de hipoalimentación.

\section{MATERLAL Y METODOS}

Se estudiaron 242 niffos de ambos sexos provenjentes del Area Central de Santiago. Los requisitos para ingresar al estudio fueron edad gestacional de 38 a 41 semanas, peso de nacimiento entre 2.500 y $3.850^{\circ} \mathrm{g}$ considerado ade- 
cuado a su edad gestacional según la Curva de Lubchenco ${ }^{1 \mathrm{~B}}$, examen físico nomal y lactancia exclusiva al mes de edad.

Los requisitos de selección para las madres de estos niños fueron 18 a 35 años de edad, paridad 1-3, embarazo normal, parto vaginal, salud y estado nutricional nomal y hemoglobina superior a $10 \mathrm{~g} \%$ al día 30 postparto. Se excluyeron quienes recibian drogas o anticoncepción hormo. nal. Para evaluar el estado nutricional se usó el índice peso talla y la tabla de peso aceptable del I.C.N.N.D. ${ }^{19}$. Sólo se ingresaron mujeres que tenían interés en lactar por un período prolongado.

Las madres fueron invitadas a participar en este estudio en la matemidad del Hospital Paula Jaraquemada recibiendo en ese momento las instrucciones verbales y escritas para amamantar en libre demanda. Los niffos y sus madres fueron citados a 3 controles en el primer mes de vida (dias 7,20 y 30 aprox.) y a controles mensuales hasta el año. Los niños se citaron a controles adicionales cuando hubo dudas sobre la suficien. cia de la lactanciá. Además se las invitó a consultar espontáneamente si tenf́an dudas acerca de la salud o el crecimiento del niño.

En cada visita, se hizo control pediátrico del niffo, examen mamario y ginecológico a la madre. Las madres recibjeron instrucciones para llenar un registro escrito de la frecuencia diaria de mamadas el que se revisó en cada control.

Se practicó examen de $\mathbf{H b}$ a las madres al día 30 postparto y a los lactantes al mes $6^{\circ}$ de vida o antes si se sospechó anemia. Los niños cumplieron el programa de inmunizaciones vigente para el Ministerio de Salud.

El estado socioeconómico de la población fue estudiado mediante el Método de Graffar ${ }^{20}$ a través de una visita domiciliaria a cargo de una asistente social.

Las madres recibieron educación sobre las ventajas de la lactancia exclusiva y se les recomendó activamente amamantar en libre demanda y sin uso de chupete o adición de agua u otros nutrientes hasta su indicación por el pediatra. En los controles se reforzo la motivación y se las felicitó por el buen progreso del niño.

Cuando se observó una curva ponderal normal, se indicó pecho exclusivo en libre demanda durante los primeros 6 meses agregando almuerzo, jugos y agua al $7^{\circ}$ mes y comida al $9^{\circ}$ mes. Los niños recibieron diariamente gotas vitamínicas (vit a $1.500 \mathrm{U}$., vit $\mathrm{D} 600 \mathrm{U}$. y vit C $50 \mathrm{mg}$ ), junto a una de las mamadas. Cuando se hizo diagnóstico de hipogalactia, se indicó leche en fórmula adecuada a la edad y peso y alimentos no lácteos según la edad. Para obtener aceptación de la leche no matema en niffos mayores de 5 meses, se sugirio a la madre darle en taza o como postre, como también establecer horario de alimentación al pecho, después de cada mamadera.

Para evaluar la suficiencia de la lactancia se usó como criterio fundamental la curva de peso del nifio, su peso absoluto y la mantención del nino en su percentil de alza ponderal y de talla. Como referencia se utilizó el estándar de Boston para ninos hombres ${ }^{21}$, copia del cual se incluyó en cada ficha. También se analizó, concediéndosele importancia secundaria, la apreciación materna de que el niño "queda con hambre", las características de las deposiciones del niño y el examen mamario de la madre. Se diagnosticó hipogalactia al encontrar falla ponderal en 2 o mas controles, analizando individualmente cada caso.

Se llamó lactancia exclusiva o absoluta al período en que la leche materna fue el único alimento hasta el $6^{\circ}$ mes y el único aporte lácteo hasta el $12^{\circ}$ mes. Se llamó lactancia parcial o suplementada al período en que los niños recibie. ron suplemento lácteo además del pecho. Se llamó lactancia suspendida a los casos en que el niño dejó el pecho materno por completo.

Para la confección de las curvas de crecimiento que se muestran en este estudio, sólo se consideraron los casos en lactancia absoluta ex. cluyendo el peso correspondiente al mes en el cual se hizo el diagnóstico de hipogalactia.

\section{RESULTADOS}

Se enrolaron en este estudio 242 niños que estaban en lactancia exclusiva al dia 30 de vida: $127(52 \%)$ eran hombres y $115(47,5 \%)$ eran mujeres.

Las características de sus madres eran (X \pm S.D.): edad $23 \pm 3$ affos, peso $56 \pm 6 \mathrm{~kg}$, Hemoglobina (Hb) $13,4 \pm 1 \mathrm{gr}$. El índice peso--talla estuvo bajo $90 \%$ en $9,7 \%$ de etlas y sobre $109 \%$ en $20 \%$; sólo hubo 4 madres bajo $80 \%$.

La condición socioeconómica se evaluó en 190 casos. E1 93,5\% de las familias estaba en el nivel socioeconómico medio-bajo y bajo, correspondiendo el $48,8 \%$ al Grupo III y el $44,7 \%$ al grupo IV de la clasificación de Graffar. Sólo el $4,2 \%$ fue clasificado en el Grupo $v$ que corresponde a la situación de extrema pobreza.

En la Figura 1 se aprecia la evolución de la lactancia en el grupo estudiado. Al $6^{\circ}$ mes de edad el $59 \%$ de los niños estaba en lactancia exclusiva y el $24 \%$ en lactancia parcial, habiéndose producido el destete en el $10 \%$ de los casos. En 
e) restante $7 \%$ de los casos se interrumpió el seguimiento antes del $6^{\circ}$ mes mientras estaban aún en lactancia. Al afio de edad, el $44 \%$ de los niños estaba recibiendo alimentación al pecho y el $15 \%$ del total permanecia en lactancia exclusiva. El número promedio de mamadas por cada 24 hrs. durante lactancia exclusiva fue 10.9 en el primer mes y 9,3 en el $6^{\circ}$ mes.

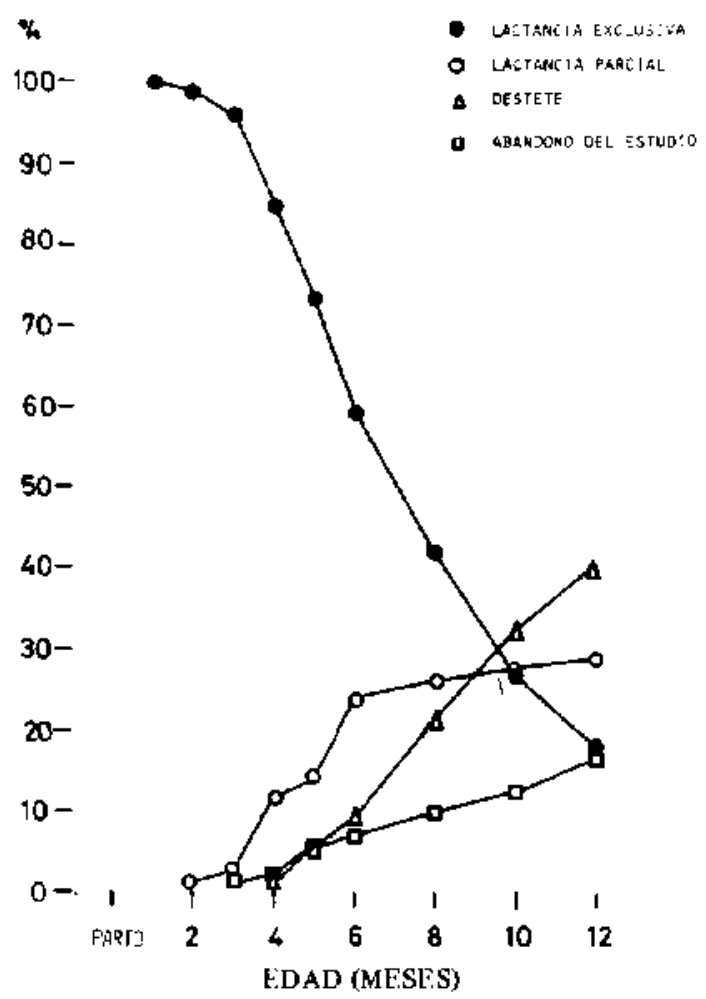

Figura 1. Distribución de los niños de acuerdo al tipo de lactancia y a la edad.
La causa más frecuente para indicar suplemento fue el diagnóstico de hipogalactia que se hizo en $66(27 \%)$ de los casos entre el $2^{\circ}$ y $6^{\circ}$ mes y en $74(30.7 \%)$ en el $2^{\circ}$ semestre. También se indicó suplemento en 14 casos en que la madre se embarazó, en 5 casos por enfermedad intercu. rrente de la madre o del lactante y en 5 casos por trabajo materno. En otros 19 casos el suplemento fue administrado por decisión de la madre a pesar de que el niño tenía un crecimiento adecuado en los controles previos. En esos casos las razones aducidas fueron la impresión de que el niño tenja hambre, el cansancio materno por las mamadas frecuentes o el consejo de familiares.

La Tabla 1 muestra los percentiles y promedios de peso observados para cada mes para el conjunto de niños de ambos sexos hasta el año de edad. El percentil 50 de esta serie es ligeramente superior al percentil 50 de la serie de Boston desde el $3^{\circ}$ al $6^{\circ}$ mes de edad.

En la Figura 2 se observa la evolución de los incrementos mensuales de peso durante el período de lactancia exclusiva. Se observan incrementos mensuales superiores a $700 \mathrm{gr}$. en los primeros 3 meses e inferiores a $600 \mathrm{gr}$. en el $5^{\circ}$ y $6^{\circ}$ mes.

La proporción de casos en lactancia exchusiva al $6^{\circ}$ mes postparto fue $72 \%$ en el subgrupo de niños y $57 \%$ en el caso de las niñas. Esta diferencia es estadísticamente significativa según análisis de tabla de contingencia.

La Figura 3 muestra los percentiles 3, 50 y 97 de peso en los primeros 6 meses de vida en el grupo completo y subdividido en los niños y niñas durante lactancia exclusiva. El percentil 50 al $6^{\circ}$ mes fue $8170 \mathrm{gr}$. para los niños y $7835 \mathrm{gr}$.

Tabla 1 .

Percentlles de Pcso de Niños Chilenos en Lactancia Exclusiva (g)

Ldad

\begin{tabular}{|c|c|c|c|}
\hline$(\operatorname{mc}<\bar{s})$ & 3 & 10 & 25 \\
\hline $\begin{array}{l}\text { Naci- } \\
\text { misrto }\end{array}$ & 2665 & 2825 & 3020 \\
\hline 1 & 3420 & 3660 & 3845 \\
\hline 2 & 4290 & 4610 & 4875 \\
\hline 3 & s1) $(00$ & $53 \geqslant ?$ & 5620 \\
\hline 4 & 5712 & 59901 & 6420 \\
\hline 5 & 6240 & 6570 & $? 000$ \\
\hline 6 & 6710 & 7110 & $75 f 0$ \\
\hline 8 & 7525 & 7990 & 8420 \\
\hline 10 & 8145 & 8510 & $90 \geq 0$ \\
\hline 12 & 8815 & 9065 & 9595 \\
\hline
\end{tabular}

Percen.

tils:s

50

3200

4190

$5 ? 10$

6080

6820

7525

90135

8870

9430

9970

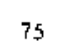

3410

4480

5570

6410

7200

7860

8381

9315

997?

10585

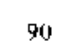

3575

4672

5885

6700

7575

8225

8870

9820

10780

11190
97

3710

4840

6100

7130

3970

8780

9335

10440

[1] 80

11550

\section{$\overline{\mathrm{X}} \pm$ D.S}

242

$3215 \pm 280$

241

$4182 \pm 395$

234

$5227 \pm 505$

212

$6057 \pm 572$

182

$6835 \pm 618$

143

$7478 \pm 658$

121

$8020 \pm 695$

82

$8927 \pm 733$

55

$9577 \pm 783$

33

$10304 \pm 776$ 
para las nifias. El peso de nacimiento y al $6^{\circ}$ mes de edad y el alza ponderal desde el nacimiento hasta los 6 meses fueron significativamente más altos en los niños que en las niñas según el test de Student.

El valor de la hemoglobina al sexto mes de vida ( \pm 1$)$ en 114 niños en lactancia exclusiva fue $11,8 \pm 0,9 \mathrm{gr} \%(\mathrm{X} \pm$ S.D.) con un rango entre 9,1 y $14,1 \mathrm{gr} \%$, habiéndose detectado sólo 3 casos con valores inferiores a $10 \mathrm{gr} \%$.

En la Tabla 2 se señala la morbilidad observada durante el 1er año de vida de los riffos mientras estuvieron en lactancia exclusiva. La incidencia de patologia fue escasa, no hubo mortalidad y sólo 4 hospitalizaciones: 2 por bronquitis obstructiva y 2 por cirugía (1 hemia inguinal y 1 torsión testicular). La patología más frecuente fue la respiratoria inchyendo, bronquitis, neumonia, laringitis, amigdalitis, otitis y estados gripales. Hubo sólo 2 casos de desnutriciôn leve.

El grupo de niños que fueron suplementados o destetados presentó un peso promedio de $7231 \pm$ 590 g y $9389 \pm 812 \mathrm{~g}$ a $\operatorname{los} 6$ y 12 meses de vida respectivamente. Ambos valores son significativa-
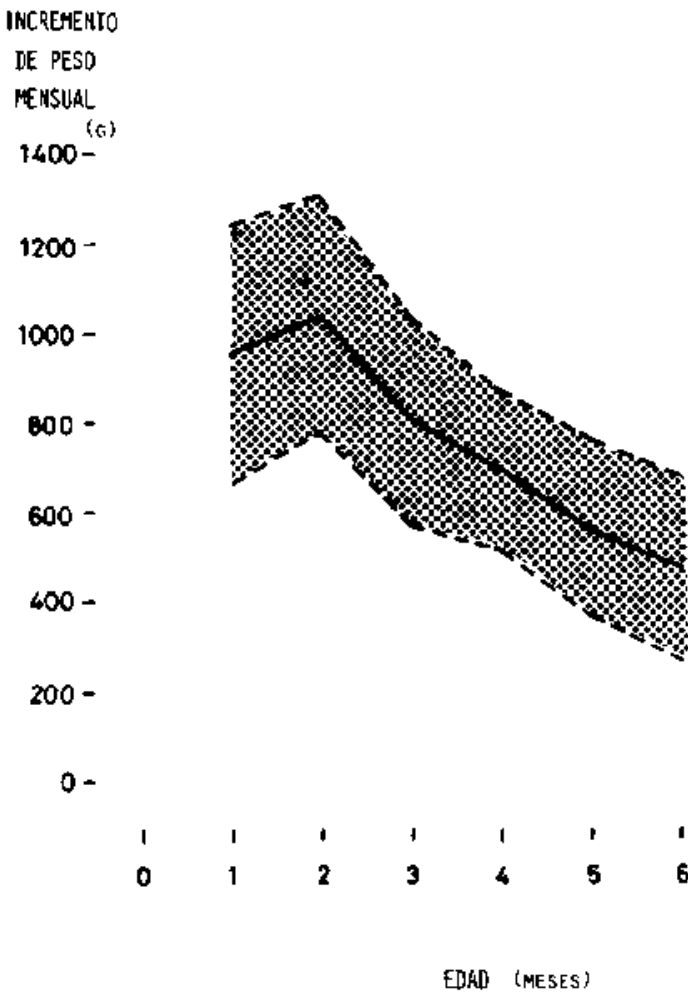

Figura 2. Incrementos mensuales de peso observados en los niños en lactancía exclusiva $(X \pm D$ D.S.). mente menores que los encontrados para el grupo en lactancia exclusiva de acuerdo al test de student. En este grupo se detectó desnutrición en 1 caso entre 81 antes de los 6 meses de edad y en 7 niffos entre 169 antes del año, lo que representa una frecuencia más alta que la del grupo en lactancia exclusiva.

Tabla 2.

Morbilidad de Niños en Lactancia Exclusiva

$$
\begin{aligned}
& \text { r.dad (meses) } \\
& \begin{array}{llll}
2 & 6 & 7 & 12
\end{array}
\end{aligned}
$$

No. de niños $\quad 241 \quad 122$

No. total de meses en laciancia cxetusiva $\quad 123 ?$

I:ntermedades (No. caros / No. episodios)

Diarsea cntera!

Desnutrición

Infeccione: Iespiratorias

Anemia ferropriva

Orros

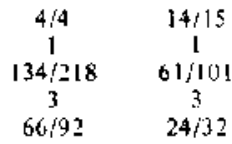

Hospitalizaciones

$3 / 3 \quad 1 / 1$

Figura 3. Percentiles de peso de los niños en lactancia exclusiva en los primeros 6 meses de edad. 


\section{DISCUSION}

Los resultados de este estudio, demuestran que es posible obtener un adecuado crecimiento de los lactantes alimentados al pecho exclusivo en los primeros 6 meses de vida, bajo circunstancias controladas y tomando como referencia curvas de peso de países desarrollados $21-22$. Este adecuado crecimiento se abtuvo en el $60 \%$ de los niffos que sólo recibieron leche materna hasta el $6^{\circ}$ mes de vida. En el $40 \%$ restante, el control de peso periódico fue fundamental para detectar hipogalactia oportunamente. Nuestros datos confirman las conclusiones de otros autores nacionales en cuanto a que la lactancja exclusiva es capaz de sustentar un adecuado crecimiento del nifึo 3-23-24.

Los pesos de estos ninos fueron superiores a los comunicados para niños en lactancia exclusiva de países en desarrollo ${ }^{16}$. Esta diferencia puede deberse a la exigencia de un alza ponderal mínima impuesta en este estudio, lo que llevó a indicar suplementación láctea a aquellos casos donde el crecimiento era inadecuado.

En este estudio, el porcentaje de niños varones en lactancia exclusiva al $6^{\circ}$ mes fue mayor que el de niñas. También los hombres tuvieron pesos mayores. Estas diferencias fueron estadisticamente significativas y deben ser tomadas en cuenta al analizar la curva de peso de cada niño en control.

Coincidimos con otros autores $25-26$ en que no hay riesgo importante de anemia ferropriva en el nino alimentado exclusivamente al pecho; de acuerdo a nuestros resultados, en hijos de madre con $\mathrm{Hb}$ nomal y en los rangos de Peso de nacimiento (PN) estudiados, existe alta probabilidad de $\mathrm{Hb}$ normal al $6^{\circ}$ mes.

Es muy importante comparar la morbilidad de este grupo de niños con aquella de la ciudad de Santiago en el mismo período. En este estudio se observó una baja frecuencia de diarrea, hospitalización, anemia y desnutrición durante el amamantamiento exclusivo y no hubo muertes. En Santiago, en 1978, la mortalidad infantil después del 1er mes fue $25 \%$ o y el indice de desnutrición en el primer semestre fue $5,4 \%$ y de $13 \%$ en el primer año de vida ${ }^{27 \cdot 28}$. La mejot condición de salud de los niños en el estudio puede deberse parcialmente al hecho de ser un grupo de niños normales al nacer $\mathrm{y}$ al tipo de atención médica recibida, pero es necesario destacar la influencia positiva del amamantamiento, factor de reconocido valor preventivo de morbilidad, desnutrición y mortalidad $2-5-6-29$.

Nos parece que los resultados de este estudio pueden contribuir a la promoción de la lactancia materna en Chile ya que demuestran que un alto porcentaje de mujeres puede lactar en forma satisfactoria y que la evolución de los niños que amamantan es óptima durante el primer año de vida. También creemos que la motivación del equipo de salud y el control periódico de las madres y los ninoos son hertamientas indispensables para obtener estos resultados en una pobla. cjón urbana.

\section{RESUMEN}

Se describe el crecimiento de niños sanos durante el período de lactancia exclusiva. La muestra está compuesta por 242 niños de nivel socioeconómico medio-bajo y bajo que estaban en lactancia exclusiva a los 30 días de edad. El $59 \%$ de ellos estaba en lactancia absoluta al $6^{\circ}$ mes de edad y mostraba un crecimiento normal sin haber recibido suplementos lácteos o no lácteos. En el $27 \%$ de los casos se indicó suplemento lácteo por hipogaláctea $y$ en el $14 \%$ restante por otras razones como embarazo, enfermedad, trabajo o decisión materna. El incremento de peso observado durante los 3 primeros meses de lactancia exclusiva fue mayor que el observado posteriormente. Un mayor porcentaje de niños que de nittas estaba en lactancia exclusi. va al $6^{\circ}$ mes y los hombres tuvieron un incremento de peso superior a las mujeres al $6^{\circ}$ mes de edad. Se presentó una baja frecuencia de patología gastrointestinal, desnutrición y hospitalizaciones.

Se concluye que la leche materna es suficiente alimento para el lactante hasta el $6^{\circ}$ mes de edad si es producida en cantidad adecuada. Se recomienda la lactancia exclusiva en libre demanda asociada al monitoreo de peso hasta el $6^{\circ}$ mes más que la prescripción rutinaria de suplementos precoces.

\section{AGRADECIMIENTOS}

Los autores agradecen a las autoridades de la Maternidad del Hospital Paula Jaraquemada y del Area Central de Salud por las facilidades otorgadas, al International Development Research Centre, Canadá por el financiamiento de esta investigación y al Centro Nacional de la Familia, Chile por el apoyo institucional.

\section{REFERENCIAS}

1 Comiemporary patterns on breastfeeding. Report on the WHO Collaborative Study on Breastfeeding. Worid Health Organization. Geneva 1981. Office of Publications. World Health Organization. p. $22-23$ and $32-46$. 
${ }^{2}$ Mc Cann M., Liskin L., Piotrow' P., Rinehart W. and

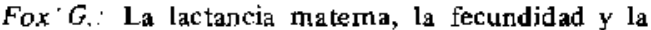
planificación fantiliar. Population Reports. Serie $J$. No 24, 1982 .

3 Pereda $C$. Rodriguez $J, y$ col. Diagnóstico de situa ción de la lactancia materna en cuatro regiones de salud de Chile. Cuadernos Médicos Sociales XXIII: 13,1979 .

4 Jelliffe D.B.: World trends in infant feeding. Aro. J. Clin. Nut. 29: 1227, 1976.

${ }^{5}$ Plenk S.J, and Milanesi M.L.: Infant feeding and infant mortaljty in miral Chile. Bull. Whd. HIth. Org. 48: $203,1973$.

6 Cunningham A.S. Morbility in breastfed and artificially fed intsnts. J. Pedjatr. 90: 726, 1977.

' Kielmen A.A., McCord C.: Weigh t-for-age as an index of risk of deth in children. Lancet I: 1247 . 1978.

${ }^{8}$ Lamm R.D., Delaney B.S, and Duryer J.T.: Economy in the feeding of infants. Pediatric Clinics of North America 24: 71, 1977.

Jackson. Long-term consequences of suboptimal mutritional practices in early life. Ped. Clin. North Am. 2979: 63, 1977.

10 Gonzalez N., Hertramph E., Mardones F., Rosso P. y Verdugo $C$ : Evaluación preliminar del programa de lactancia materna. Rev. Chil. Pediatr. 54: 36, 1983.

1 Vera L.F., Anderson R., Cruz A., Jeldres, V., Jimé. nez J., Patri A.: Influencia de la actitud médica en la duración de la lactancia natural. Rey. Pediatria 18: $77,975$.

12 Valiente $S$ : Lactancia como problema de salud pública en Chile. Symposium Lactancia. XIV Congreso Chileno de Obste tricia y Ginecologia. Santiago 7-12 Noviembre, 1971.

13 Morin P.: Lactancia matema en el Area Oriente de Santiago. Rev. Chil. Pediat. 50: 71, 1979.

14 Mardones F.: Marco histórico para el análisis de las causas del descenso en la práctica de la lactancia matema: Rev. Chil. Pediatr. 53; 607, 1982.

15 Taller Subregional; Lactancia matema y nutrición matemo-infantil. Ministerio de Salud de Chilef OPS/OMS. Noviembre 1980. Santiago, Chile.

16 Waterlow J.C., Ashworth A., Griffiths M.: Faltering in infant growth in less-developed countries. Lancet IJ: 1176,1980 .

17 Monckeberg $F$.: Lactancía matema corta: alto riesgo en paises subdesarrollados, Apartado Docente 3/76. Nutrición y Tecnología dde Alimentos. p. 25 , NES, 1976.

18 Lubchenco L.O., Hansman Ch., Dressier M., Boyal $E$.: Intrauterine growth as estimated from live born weight data at 24 to 42 weeks of gestation. Pediatrics $32: 793,1963$.

19 Interdepartamental Committee on Nutrition for National Defense: Manual for Nutrition Surveys. 2nd ed. Washington, D.C.: ICNND, 270, 1973.

20 Graffar $M_{1}$ : Une methode de classification sociale d'echantillons de population. Courrier 6: 455, 1956.

21 Vaughan V.C.: Growth and development of the infant and the child. In: Texbook of Pediatrics, 8 edition, Phidadelphia (ed) Nelson W.E. W.B. Saunders Company. 1964, p. 42.

22 Sempe M., Roy M.P. et Pedron G.: Croissance en taille at en poids de garcons de la naissance a 17 ans. In: Pédiatric Sociale, Paris. (ed) Mande R., Nasse N., Manciaus M. Flammarion Médicine-Sciences. 1972.

23 Repetto G.: Influencia de la lactancia materna en el niño. Rev. Pediatría 16: 119, 1973.

24 López I., Cabiol C., Arcuch S., Rivera E, y Vargas S: Lactancis materna y aumento de peso en el primer año de vida. Rev. Chil. Pediatr, $51: 473,1980$.

25 Picciaro M.F. Deering R.H.: The influence of feeding regimens on iron status during infancy. Am. J. Clin. Nut. 33: 746, 1980.

26 Simes M.A., Vuori E, and Kuitunen P.: Breast milk iron $-A$ declining concentration during the course of lactation. Acta Paediatr. Scand. 68: 29, 1979.

27 Ministerio de Salud. Anuario 1978. Atenciones y Recursos 1979. (ed) Ministe rio de Salud de Chile.

28 Consejo Nacional para la Alimentación y Nutrición, Ministerio de Salud de Chile: Chile, estadisticas básicas en alimentación y nutrición, 1969-1978. Santiago, Chile, 1980.

29 Lopez I., Cabiol C., Arcuch S., Rivera E., Vargas S. y San Miguel A.: Lactancia matema y morbilidad por diartea $y$ desnutrición en el primer año de vida. II Parte. Rev. Chil. Pediatr. 53: 162, 1982. 